\title{
Comparison of the accuracy of curve-fitting methods for the determination of gas permeability parameters of sheet polymer samples
}

\author{
Tibor Guba ${ }^{\mathrm{a}}$,Zoltán Filus ${ }^{\mathrm{a}, \mathrm{b}}$, Károly András Simon ${ }^{\mathrm{a}}$, Gábor Szabó ${ }^{\mathrm{a}}$, Zoltán Bozóki ${ }^{\mathrm{c}, \mathrm{a}}$
}

${ }^{a}$ Department of Optics and Quantum Electronics, University of Szeged, H-6720 Szeged, Dóm tér 9, Hungary

${ }^{b}$ Hilase Development, Production, Service and Trading Ltd. H-6727 Szeged, Irinyi J. u. 1, Hungary

${ }^{c}$ MTA-SZTE Research Group on Photoacoustic Spectroscopy H-6720 Szeged, Dóm tér 9, Hungary

\begin{abstract}
Accuracy of the gas permeability parameters (GPPs), i.e. solubility, diffusivity and permeability deduced from permeation measurements is investigated in case of homogeneous polymer sheet samples. The widely used time-lag method (TLM) and the recently introduced full curve-fitting method (FCFM) are compared on simulated and on measured permeation curves artificially distorted in various ways in order to mimic potential deficiencies of permeation measurements. Accuracy of the methods is defined as the relative deviation between the calculated and the real GPPs, i.e. those which are deduced from the distorted and the original, non-distorted curves, respectively. The following distortions have been applied: temporal truncation of the permeation curves, increasing the noise level of the measurement and shifting the permeation curve either along the concentration or the time axis. (The latter two transformations correspond to an unnoticed background shift in the readings of the concentration detection unit and an uncertainty in the actual inception of the permeation process, respectively). While all these distortions mimic realistic deficiencies of permeation measurements, the last one is relevant only in case of fast permeation processes through highly permeable membranes. For all but the last transformation FCFM has been found to yield more accurate GPPs than TLM.
\end{abstract}

Keywords: permeability measurements, diffusivity, solubility, permeability, non-linear curvefitting, chi-square analysis 


\section{Introduction}

Gas permeation parameters (GPPs), i.e. diffusivity $(D)$, solubility $(S)$ and permeability $(P)$ of rubber and polymer membranes are crucial material properties whenever thin flexible materials are used to isolate gases from their environment (e.g. rubber tyres, flexible gas pipes, etc.). There are four mathematical equations describing the gas permeation process in case of typical experimental arrangements. Two of them are the Fick's laws [1]:

$$
\vec{J}(\vec{x}, t)=-D \cdot \nabla C(\vec{x}, t)
$$

and

$$
\frac{\partial C(\vec{x}, t)}{\partial t}=D \cdot \nabla^{2} C(\vec{x}, t)
$$

where $C(\vec{x}, t)$ is the concentration and $\vec{J}(\vec{x}, t)$ is the current density of the permeating molecules. The third equation is Henry's law: if the partial pressure of the analysed component in the gaseous phase is $p(\vec{x}, t)$, then:

$$
C\left(\vec{x}_{0}, t\right)=S \cdot p\left(\vec{x}_{0}, t\right)
$$

where $x_{0}$ denotes the coordinates of the contact points between the sample and the gaseous phase. The fourth equation gives the correlation between the GPPs [1]:

$$
P=D \cdot S
$$

In one of the simplest experimental arrangements a homogeneous plane membrane sample isolates the two chambers of the permeation cell. One of them (the so called source chamber) contains the permeating gas with a constant and high concentration (typically $C=100 \%$ ), while in the other one (in the so called receiving chamber) the concentration of the permeating molecules is zero at the start of the permeation process. In this case all equations are one-dimensional, the current density vector is perpendicular to the surface of the membrane, and if the thickness of the membrane is $l$, then the time dependent magnitude of the flux through the membrane (hereinafter referred to as permeation curve) can be given as [1]:

$$
|\vec{J}(t)|=\frac{P p}{l}\left[1+2 \cdot \sum_{n=0}^{\infty}(-1)^{n} \cdot e^{-\frac{n^{2} \pi^{2} D t}{l^{2}}}\right]
$$

In this experimental arrangement the solution of the inverse problem, i.e. the determination of the GPPs from the measured permeation curve, is relatively simple at least under ideal measurement conditions by using curve-fitting methods described in detail in the 
Appendix. One of these methods is the time-lag method (TLM), in which only the late, steady-state part of the permeation curve is fitted by a line and GPPs are calculated from the parameters of this line. It is a simple method which can be used even without a computer. However, it is not robust enough against measurement uncertainties and it does not indicate the possible unreliability of the deduced permeation parameters. Recently a full curve-fitting method (FCFM) was shown to be an alternative of TLM [2]. Here we use a slightly modified version of this method, by fitting the flux curve itself (see Appendix) rather than the concentration (i.e. the integrated flux). Up to now these two methods have not yet been compared systematically. Obviously, in the ideal case, when Equation 5 accurately describes the permeation process, both methods yield the GPPs characteristic for the studied sample. However, when the permeation curve is distorted, i.e. Equation 5 can be used only as an approximation, the deduced GPPs can deviate from the true material parameters. Some possible causes of the distortion of the permeation curves are:

- A permeation measurement always has a finite measurement time, which must be kept as short as possible for the sake of saving time and costs. For TLM the measurement can be terminated only after the steady-state part of the permeation process is reached (typically after a period of five times the characteristic "time-lag" time). On the other hand, FCFM offers the possibility of reducing measurement time by terminating the measurement sooner than in case of TLM. However, a method is needed to verify that the measurement is not finished prematurely, i.e. GPPs deduced from the truncated measurement curve are sufficiently close to the real GPPs values.

- During permeation experiments the concentration measurement is always loaded with noise. The standard deviation of this noise depends on many factors such as the type of the concentration measuring instrument, the permeation properties of the measured sample, etc. One can expect an increase in the difference between the real and the estimated GPPs with decreasing signal to noise ratio.

- Even when it is properly calibrated initially, there might be a temporal variation in the calibration parameters of the concentration measuring instrument. In most cases the offset (i.e. the signal in case of zero concentration) is subject to change, which can falsify the results of the measurement. This offset variation can be caused, for example, by spontaneous gas or vapour emission from the sample. One typical example is the emission of the plasticizing component. 
- Due to the finite response time of the permeation measuring system there is some uncertainty in the exact inception of the permeation process. The relevance of this uncertainty increases as the permeation process becomes faster (i.e. for highly permeable membranes).

Obviously there are other possible reasons why the permeation curves can be distorted, and consequently the deduced GPPs can deviate from their true values. In order to compare the robustness of the two curve-fitting methods, accuracy of the deduced GPPs is investigated at various degrees of different distortions for the cases listed above.

\section{The permeation curves}

\subsection{Measured permeation curves}

The experimental curves are the results of our earlier works where the concentration of the molecules permeating through the membrane is measured by using the laser based photoacoustic detection system in a carrier gas flow arrangement [3]. Fluxes through the membrane are calculated by multiplying the measured concentrations with the carrier gas flow rate and by dividing the results with the area of the measured membrane sample. Table 1 lists the measurement conditions and the results of the numerical evaluation of the measured curves, i.e. the fitted $D, S$ and $P$ parameters. It must be noted that for these permeation curves the numerical values of the GPPs are practically the same regardless of using TLM or FCFM.

\begin{tabular}{|l|c|c|c|c|c|c|}
\hline & \multicolumn{3}{|c|}{ Experimental parameters } & \multicolumn{3}{c|}{ Results of curve-fitting } \\
\hline Material & $\begin{array}{c}\text { Thickness } \\
{[\mathrm{mm}]}\end{array}$ & $\begin{array}{c}\text { Carrier gas flow } \\
\text { rate }\left[\mathrm{cm}^{3} / \mathrm{min}\right]\end{array}$ & $\mathrm{SNR}$ & $\begin{array}{c}D \\
{\left[10^{-12} \mathrm{~m}^{2} / \mathrm{s}\right]}\end{array}$ & $\begin{array}{c}S \\
{\left[10^{-6} 1 / \mathrm{Pa}\right]}\end{array}$ & $\begin{array}{c}P \\
{\left[10^{-17} \mathrm{~m}^{2} / \mathrm{s} / \mathrm{Pa}\right]}\end{array}$ \\
\hline Polyethylene & 0.14 & 400 & 19 & 0.31 & 58 & 1.8 \\
\hline Natural rubber & 0.70 & 250 & 50 & 39 & 2.4 & 9.5 \\
\hline
\end{tabular}

Table 1. Experimental parameters and results of curve-fitting for photoacoustically measured samples. Measurements are performed at $23{ }^{\circ} \mathrm{C}$ with a sample area of $38 \mathrm{~cm}^{2}$ and the permeating gas is methane. SNR stands for signal to noise ratio of the measurement determined at the plateau of the permeation curve.

\subsection{Simulated permeation curves}

As a first step, noiseless permeation curves with various $D$ and $S$ values are generated by using Equation 5 in the time interval between $t=0$ and $t=15$ TL (TL stands for the timelag parameter, see Appendix). Next, randomly generated noises having an average value of 0 and a standard deviation which is 0.005 times the maximum flux value (i.e. the steady-state 
flux which is reached at the late part of the permeation process) are added to each permeation curve. The main parameters of these simulated permeation curves are listed in Table 2. In order to improve the reliability of the statistical analysis, for each $D$ and $S$ values three permeation curves are generated by repeating the noise generation process three times.

\begin{tabular}{|c|c|c|c|c|c|}
\hline $\begin{array}{c}D \\
{\left[10^{-11} \mathrm{~m}^{2} / \mathrm{s}\right]}\end{array}$ & $\begin{array}{c}S \\
{\left[10^{-6} 1 / \mathrm{Pa}\right]}\end{array}$ & $\begin{array}{c}P \\
{\left[10^{-17} \mathrm{~m}^{2} / \mathrm{s} / \mathrm{Pa}\right]}\end{array}$ & $\begin{array}{c}\text { Time-lag } \\
{[\mathrm{s}]}\end{array}$ & $\begin{array}{c}\text { Maximal flux } \\
{\left[10^{-15} \mathrm{~m}^{3} / \mathrm{s}\right]}\end{array}$ & $\begin{array}{c}\text { Noise } \\
{\left[10^{-15} \mathrm{~m}^{3} / \mathrm{s}\right]}\end{array}$ \\
\hline 10 & 0.5 & 5.0 & 1667 & 19200 & 96 \\
\hline 3.0 & 0.8 & 2.4 & 5556 & 9200 & 46 \\
\hline 1.0 & 1.0 & 1.0 & 16670 & 3800 & 19 \\
\hline 0.3 & 1.5 & 0.45 & 55560 & 1740 & 8.7 \\
\hline 0.1 & 2.0 & 0.20 & 166700 & 760 & 3.8 \\
\hline
\end{tabular}

Table 2. List of GPPs of the simulated permeation curves, the calculated time-lag parameters, the maximum flux at the late part of the permeation curve (i.e. at its plateau) and the standard deviation of the added noises (which is 0.005 times the flux value at the plateau of the permeation curve).

\subsection{Artificial distortion of the permeation curves}

In order to mimic possible discrepancies of the permeation measurements the following transformations were performed on measured and simulated permeation curves. Transformation parameters (TPs) representing the degree of distortion have been introduced as follows:

- Truncation: Distorted permeation curves are generated by removing the late parts of the permeation curves in various lengths. TP is defined as the duration of the nontruncated part of the permeation curve normalized with the time-lag parameter (i.e. $t_{\max } / \mathrm{TL}$, for the definition of the time-lag parameter see Appendix).

- Added noise: Random noises having a normal distribution with an average value of 0 and various standard deviations are added to both the measured and the simulated curves. TP is defined as the standard deviation of the added noise divided by the maximum flux value reached at the plateau of the permeation curve (i.e. 1/SNR, the inverse of the signal-to-noise-ratio).

- Vertical shift: Permeation curves are shifted along the vertical (flux) axis into positive direction. TP is defined as the vertical shift $(\Delta J)$ normalized by the maximum flux value (i.e. $\left.\Delta J / J_{\max }\right)$. 
- Horizontal shift: Permeation curves are shifted along the horizontal (time) axis. TP is defined as the horizontal shift $(\Delta t)$ normalized with the time-lag parameter (i.e. $\Delta t / \mathrm{TL}$ ).

\subsection{Evaluation of the numerical fitting methods}

The accuracy of the diffusivity and permeability parameters ( $\Delta D$ and $\Delta P$, respectively) deduced by either of the fitting methods is defined as:

$$
\Delta D=\frac{D_{d}-D_{o}}{D_{o}} \quad \Delta S=\frac{S_{d}-S_{o}}{S_{o}}
$$

where $d$ and $o$ are the subscripts of GPPs which have been calculated either from the distorted or the original, undistorted curves, respectively.

\section{Results}

Figures 1 to 4 show the accuracy of the deduced $D$ parameters as a function of the various TP values. Parameters deduced by the TLM and the FCFM methods are marked with squares and triangles, respectively. Open and closed symbols represent the results of the evaluation of measured and simulated curves, respectively.

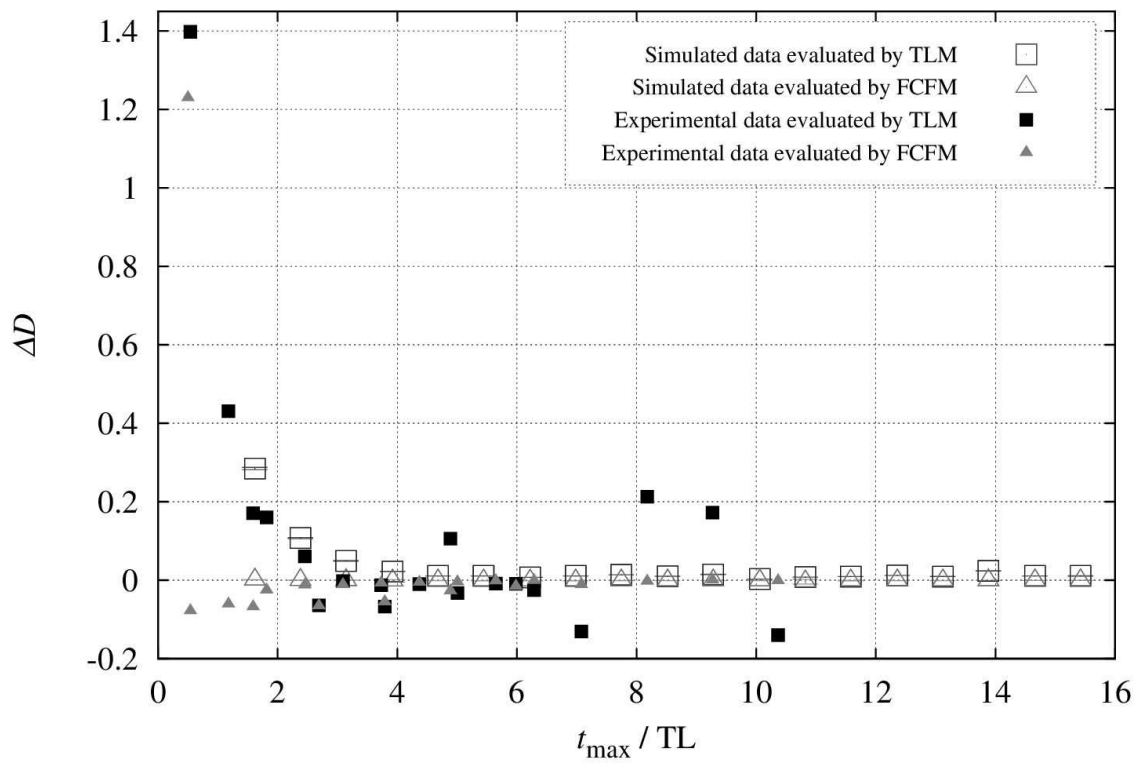

Figure 1. Accuracy of the diffusivity parameter at various degrees of truncation of the permeation curve. 


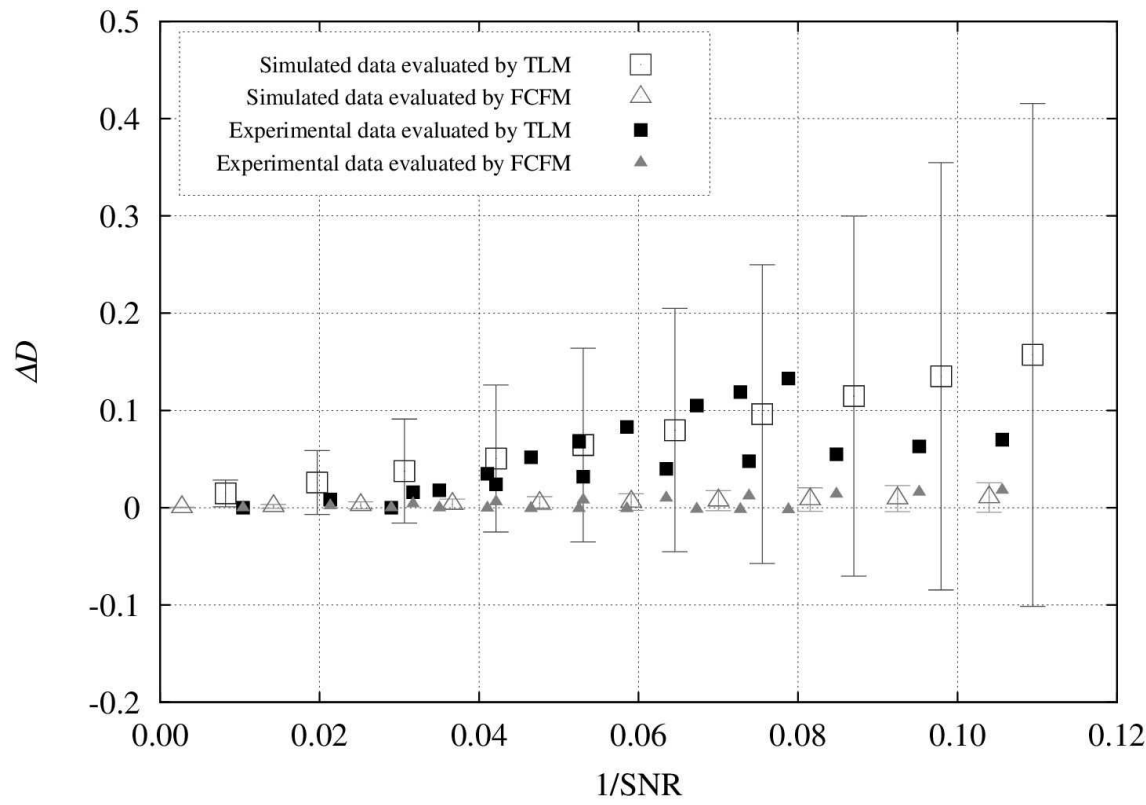

Figure 2. Accuracy of the diffusivity parameter at various degrees of noise added to the permeation curve.

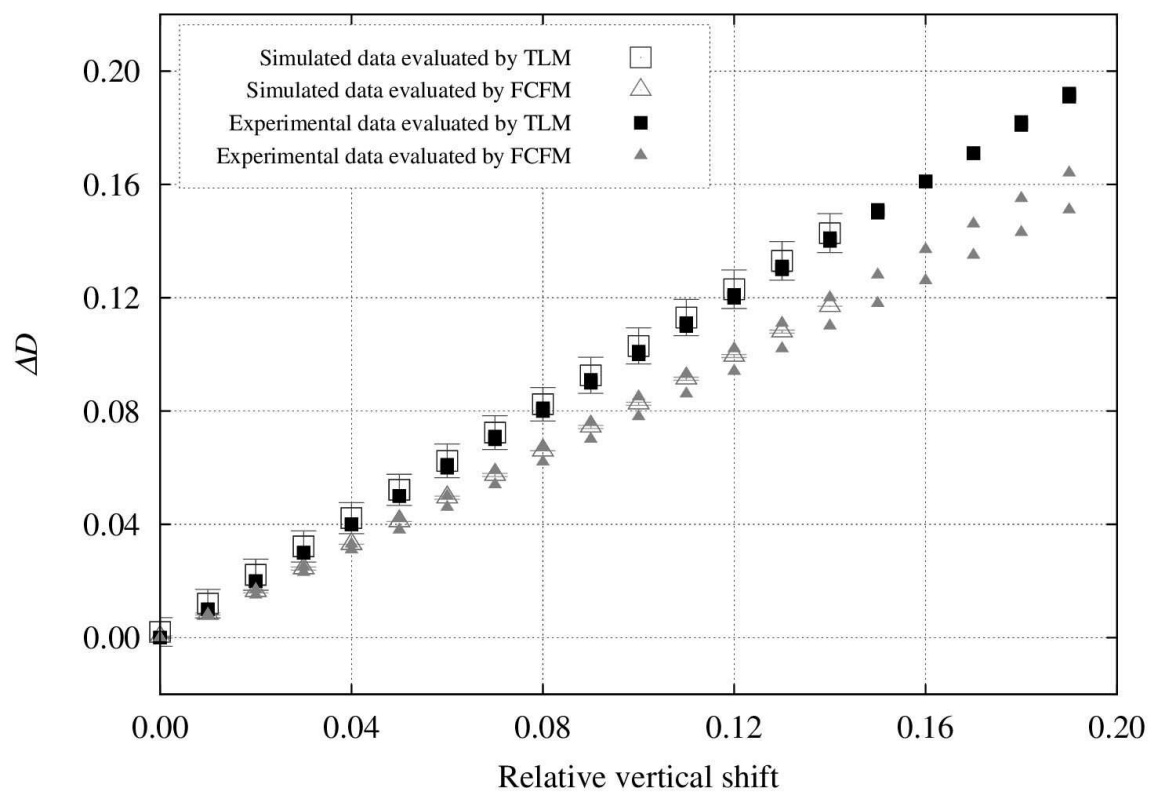

Figure 3. Accuracy of the diffusivity parameter at various degrees of vertical shift (i.e. along the flux axis) of the permeation curve. 


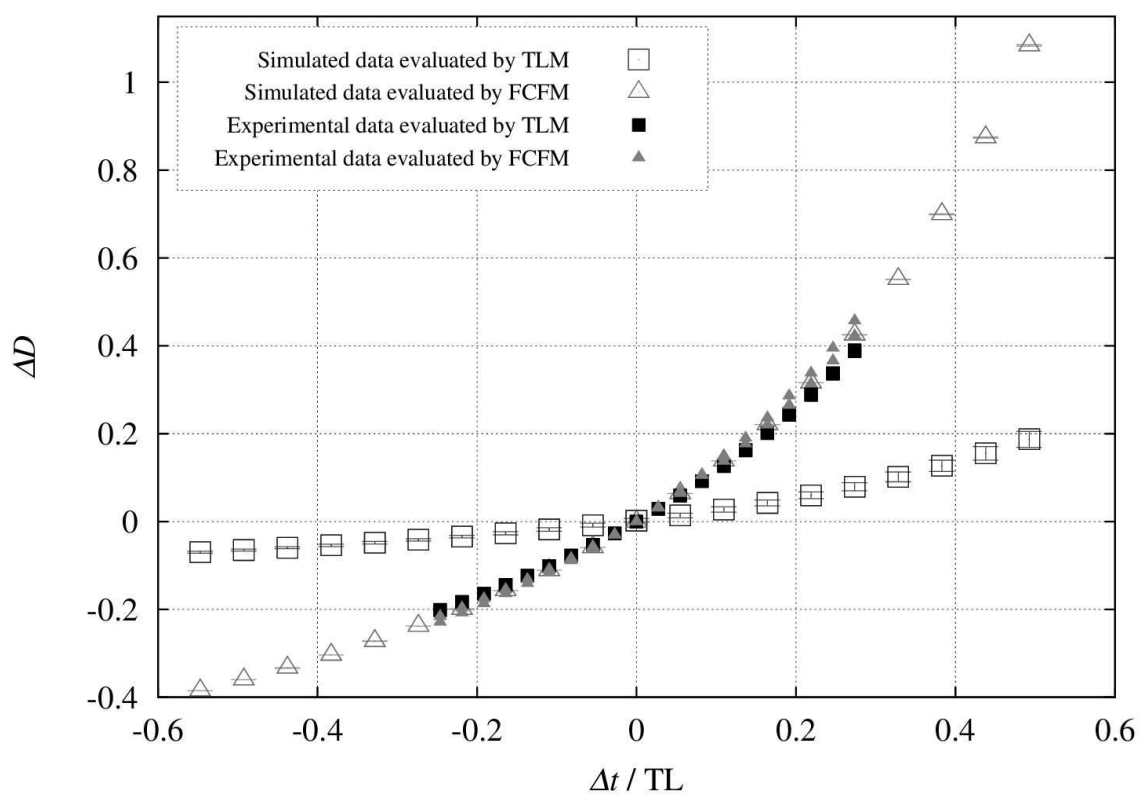

Figure 4. Accuracy of the diffusivity parameter at various degrees of horizontal shift (i.e. along the time axis) of the permeation curve.

\section{Discussion}

In order to judge the practical usefulness of the results presented above one has to first specify the maximum value of acceptable inaccuracy in the deduced GPPs and then investigate whether the transformation corresponding to this inaccuracy is feasible under realistic measurement conditions. In the following the largest acceptable inaccuracy in GPPs is assumed to be $20 \%$. However, by using Figures 1 to 4 , different tolerance levels can be examined too.

Truncation of the permeation curves is a transformation worth studying as one can save considerable measurement time and thus money by finishing the permeation measurement sooner, even if a certain level of inaccuracy may result in the deduced GPPs. As it can be seen in Figure 1, by using FCFM on measurements with high SNR, diffusivity parameters can be calculated with acceptable accuracy even when the length of the permeation curves is only twice that of the TL parameter. Furthermore, at the same degree of truncation FCFM always yields more accurate diffusivity parameters than TLM, for which there are at least two reasons. First, TLM heavily relies on the late part of the permeation curve, and truncation makes this part of the curve disappear. However, for FCFM the early and the late parts of the permeation curve have equal importance. Secondly, before performing TLM the flux curve has to be integrated, and this integration actually transforms 
measurement noises into low frequency fluctuations typically with increased amplitude. Therefore, the truncation of these large fluctuations might generate large uncertainties in the TLM results. This latter effect of the integrated noise is more emphasized for permeation curves with only moderate signal to noise ratios, as it can be seen in Figure 1 for diffusivity parameters calculated by TLM from the experimental curves. Indeed, in this case relatively large inaccuracies appear already at large $t_{\max } / \mathrm{TL}$ values, i.e. with minor degrees of truncation. Furthermore, $S$ and $P$ have also been found to follow similar curves (not shown). The only difference is that at a low value of the $\Delta t / \mathrm{TL}$ parameter TLM systematically underestimates the true $S$ and $P$ values.

In case of measurements with increased noise levels FCFM again outperforms TLM (see Figure 2). Since permeation curves with low signal to noise ratios are measured quite often, this result has its importance too. Furthermore, as it can be seen in Figure 2, the diffusivity parameter can be reliably determined by using FCFM even if SNR $=10$. For the $S$ and $P$ parameters similar curves are measured (not shown).

Similarly to both transformations discussed above, uncontrolled and uncorrected background shift has a similar effect on the deduced diffusivity parameter, i.e. once again those calculated by FCFM is less affected by this discrepancy. Based on our general measurement experience, background shifts as high as $20 \%$ of the plateau value of the permeation curve can easily occur in case of thick samples with low permeability, so all data points shown in Figure 3 are relevant. As far as solubility is concerned, unfortunately it is hard to deduce any conclusion from our results, because for some reason in this case there is a considerably deviation between simulation and measurement.

Finally, regarding the temporal shift of the permeation curve it is worth examining what constitutes a realistic uncertainty in the inception of the permeation process. Depending on the experimental conditions (i.e. the flow rate of the feed and the carrier gas, the volume of the source and the receiving chamber of the permeation cell and the volume of the gas concentration measuring unit) this uncertainty can be in the 10 seconds range, while the timelag for thin samples with high diffusivity can be one minute or less, i.e. $\Delta t / \mathrm{TL}$ can be as high as 0.2 . In Figure 4 it can be seen that the error of the diffusivity value, which corresponds to this value is more than $20 \%$ whenever FCFM is applied. Consequently the application of TLM is advantageous for highly permeable membranes. 
Based on all the discussions above it is evident that with the exception of fast permeation processes (i.e. whenever the time-lag is in the sub-minute range) it is always preferable to use FCFM for determining the GPPs rather than the widely used TLM.

\section{Conclusion}

Although permeability measurements are routinely applied in various laboratories, the accuracy of the deduced GPPs is not yet in the focus of research. The goal of the presented study is to show that improper data evaluation can lead to GPPs values showing considerable deviation from the actual material parameters on the studied sample, and that with a proper numerical method (which is FCFM in most cases) accurate GPPs can be deduced even from very noisy or truncated permeation curves. Due to the mathematical tools detailed in the Appendix, FCFM is actually as easy to perform as the routinely applied TLM.

Obviously, not only the four examined transformations may introduce inaccuracies in the permeation measurement. There are several other experimental factors too [4] such as the feed and carrier flow pattern distribution within the permeation cell, the temperature distribution within the measured sample, etc. We plan to investigate the effect of these factors on the accuracy of the deduced GPPs in a forthcoming publication.

\section{Acknowledgements}

We would like express our gratitude to József Vinkó (University of Szeged) and Mirta Benšić (Josip Juraj Strossmayer University of Osijek) for their valuable advice.

\section{References}

[1] J. Crank, "The mathematics of diffusion," 1975.

[2] Z. Filus, T. Ajtai, Z. L. Horváth, Z. Bozóki, G. Pap, T. Nagy, T. Katona, and G. Szabó, "A novel apparatus based on a photoacoustic gas detection system for measuring permeation parameters of polymer samples," Polym. Test., vol. 26, no. 5, pp. 606-613, Aug. 2007.

[3] Z. Filus, N. Tóth, G. Gulyás, T. Guba, G. Szabó, and Z. Bozóki, "Carrier gas flow arrangement based photoacoustic detection method for measuring gas permeability of polymer membranes," Polym. Test., vol. 32, no. 6, pp. 1099-1104, Sep. 2013. 
[4] A. Verwolf, S. O. Farwell, Z. Cai, and P. Smith, "Performance-based design of permeation test cells for reliable evaluation of chemical protective materials," Polym. Test., vol. 28, no. 4, pp. 437-445, Jun. 2009.

[5] A. R. Chouikha, "On Properties of Elliptic Jacobi Functions and Applications," J. Nonlinear Math. Phys., vol. 12, no. 2, pp. 162-169, Jan. 2005.

[6] W. H. Press, S. A. Teukolsky, W. T. Vetterling, and B. P. Flannery, Numerical Recipes 3rd Edition: The Art of Scientific Computing, vol. 1. 2007.

[7] R. Andrae, T. Schulze-Hartung, and P. Melchior, "Dos and don'ts of reduced chisquared," p. 12, Dec. 2010.

[8] R. Fielding, "Determination of small (<30 seconds) diffusional time lags in permeation experiments," Polymer (Guildf)., vol. 21, no. August 1979, pp. 1979-1981, 1980. 


\section{Appendix}

\section{A.1. Transforming the permeation curve into a dimensionless form}

In order to rewrite the permeation curve into a form which is easy to handle (i.e. can be repeatedly calculated many times with a short execution time, which is required during the execution of a curve-fitting algorithm) it is subservient to introduce the so called base function:

$$
f(t)=1+2 \sum_{n=1}^{\infty}(-1)^{n} e^{-t \cdot n^{2}}=\vartheta_{4}\left(0, e^{-t}\right)
$$

where $\vartheta_{4}\left(0, e^{-t}\right)$ is Jacobi's elliptical theta-function [5]. The base function has an inflection point at $t \approx 0.9$, and for large $t$ values it converges to a plateau value of one (Figure A.1.). With the help of this base function Equation 5 can be rewritten as:

$$
J(t)=\frac{P \cdot p}{l} \cdot f\left(\frac{\pi^{2}}{l^{2}} \cdot D \cdot t\right)
$$

which corresponds to expressing time and flux in the unit of $t_{i}$ and $J_{p}$, respectively, where:

$$
t_{i}=\frac{0.9 \cdot l^{2}}{D \cdot \pi^{2}}, \quad J_{p}=\frac{P \cdot p}{l}
$$

(Note that at the inflexion point the flux is $J\left(t_{i}\right)=0.24 \cdot J_{p}$ ).

In this representation each permeation curve is identical regardless of the actual $D$ and $P$ parameter values. This considerably simplifies the numerical fitting procedure (in case of FCFM) as it will be shown below. 


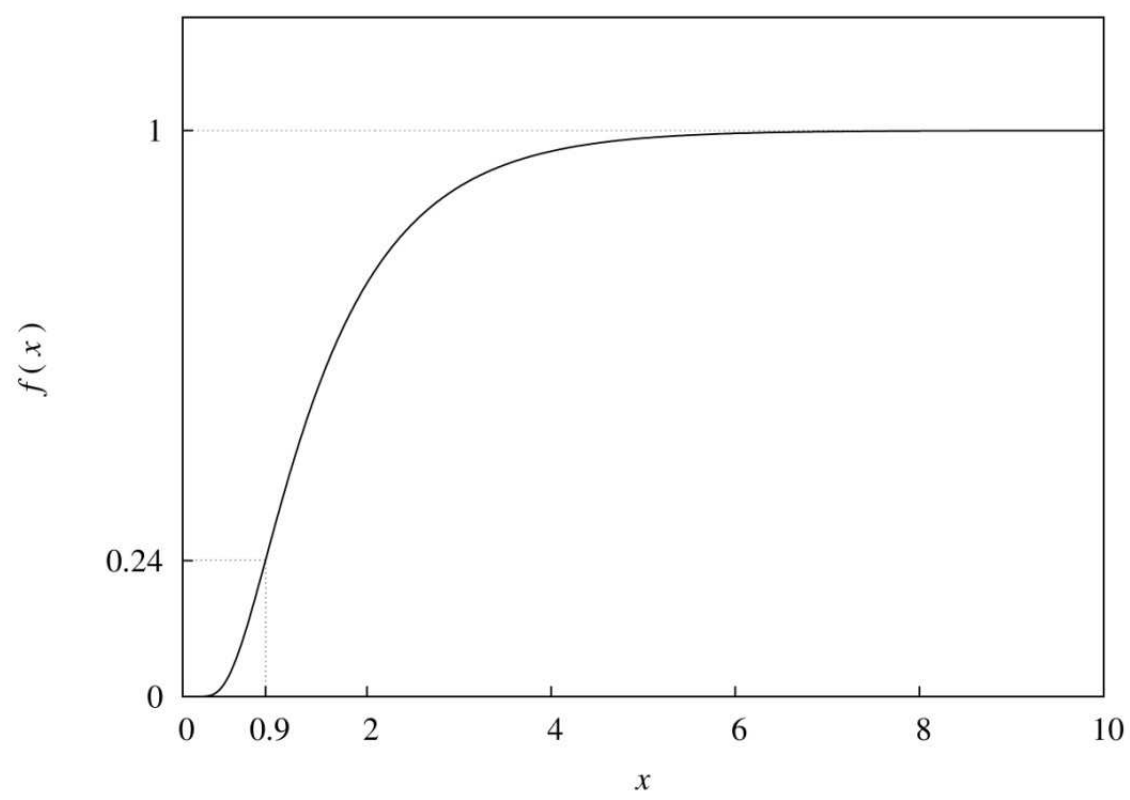

Figure A.1. The base function. See text for details.

\section{A.2. Non-linear permeation curve-fitting methods}

The model curve can be fitted numerically to the measured permeation curve by using a non-linear curve-fitting method as follows. In case the permeation curve is measured in $N$ points, and $j=1,2, \ldots N$ is the counting index of the measurement points, then the coordinates of the data points in the permeation curve can be denoted with $\left(x_{j}, y_{j}\right)$. Pearson's standard chi-square can be defined, which is the sum of the squared errors (between the measured and the model curve) divided by the independently determined standard deviation of the errors [6], [7]:

$$
\chi^{2}=\sum_{j=1}^{N}\left(\frac{y_{i}-J\left(x_{i}\right)}{\sigma_{j}}\right)^{2}
$$

This quantity follows the "chi-square" distribution with $v=N-2$ degree of freedom (because in our case there are 2 fitting parameters). The mean of this distribution also has a value of $v$. Thus the reduced chi-square quantity can be defined as:

$$
\chi_{r}^{2}=\frac{\chi^{2}}{v}
$$


In case the model curve is valid for the permeation process, the permeation parameters can be determined by using an algorithm which minimizes the value of $\chi_{r}^{2}$, and if the noise of the measurement is accurately estimated, $\chi_{r}^{2}$ has the value of approximately 1 at its minima. For general non-linear functions the Levenberg-Marquardt algorithm is used for the minimization of $\chi_{r}^{2}$. Furthermore, if the minimization procedure results in a $\chi_{r}^{2}$ value which differs significantly from 1 , this is an indication that the permeation parameters determined by curve-fitting are not valid for the measured sample.

\section{A.2.1.The time-lag method}

In order to apply the time-lag method the amount of the molecules permeated through the membrane must either be directly measured or it has to be calculated from the measured molecular current by integration. For these quantities the following equation applies [1], [8]:

$$
C(t)=\frac{A l}{V} p S\left[\frac{D t}{l^{2}}-\frac{1}{6}-\frac{2}{\pi^{2}} \cdot \sum_{n=1}^{\infty} \frac{(-1)^{n}}{n^{2}} \cdot e^{-\frac{n^{2} \pi^{2} D t}{l^{2}}}\right]
$$

It can be seen that $C(t)$ is the sum of a linear function and a series function quickly approaching 0 as time increases. The linear component can be written in the form of:

$$
C_{2}(t)=\frac{A \cdot l}{V} \cdot p \cdot S\left(\frac{D \cdot t}{l^{2}}-\frac{1}{6}\right)
$$

This is a straight line with a slope of $\frac{A p}{l V} P$, i.e. proportional to $P$.

In order to evaluate the diffusion coefficient, the interception of the time-axis is used. This point is called the time-lag $\left(t_{T L}\right)$, and it gives the second parameter of the fitted line with the equation below [1], [8]

$$
t_{T L}=l^{2} / 6 \mathrm{D}
$$

This facilitates the assessment of the parameters because after a sufficiently long measurement the only operation required to be performed is line-fitting on the latest part of the integrated permeation curve. 


\section{A.2.2. The full curve-fitting method}

In our research we used the Levenberg-Marquardt algorithm to find the least-square estimator of the permeation parameters. This algorithm is a powerful tool but its drawback is that it requires the re-evaluation of $J(t)$ for every iteration to minimize the value of $\chi_{r}^{2}$. This makes the minimum calculation procedure prohibitively long if $J(t)$ is evaluated in the form given by Equation 5. Therefore, we prefer using the base function, which has to be calculated only once, and by using the relation given in Equation A.2 it can always be transformed to $J(t)$. Furthermore, it is sufficient to calculate the base function only once at discrete $x$ values (however with a sufficiently high resolution) and in between these values it can be linearly interpolated, which substantially decreases the execution time of the non-linear curve-fitting algorithm [6]. FCFM requires an initial guess for the $P$ and $D$ parameters. For $P$ it is the average of the last $10 \%$ of the data points of the permeation curve, while the initial $D$ is calculated through $t_{i}$ by finding the time where $I \approx 0.24 I_{p}$. 